\title{
Measurement of MeV Ion Track Structure in an Organic Solid
}

\author{
R. M. Papaléo,* P. Demirev, J. Eriksson, P. Håkansson, and B. U. R. Sundqvist \\ Division of Ion Physics, Department of Radiation Sciences, Uppsala University, Box 535, Uppsala, S-751 21, Sweden
}

\author{
R. E. Johnson \\ Engineering Physics, Thorton Hall, University of Virginia, Charlottesville, Virginia 22903
}

(Received 14 December 1995)

\begin{abstract}
Radial velocities and effective ejection radii of hydrocarbon secondary ions sputtered electronically from polymer films have been experimentally determined. Using these data, the radial profiles of the energy and momentum density in an individual $\mathrm{MeV}$ ion track on a picosecond time scale are directly mapped out. [S0031-9007(96)00742-9]
\end{abstract}

PACS numbers: 61.80.Jh, 61.80.Az, 79.20.Rf

Ion tracks, formed by the interaction of energetic charged particles with matter, have played an important role in the development of modern physics. More recently, MeV-GeV ion track production has been used to modify materials in a controlled manner [1,2], being a potential tool to engineer material properties in the nanometer to micrometer scale. $\mathrm{MeV}-\mathrm{GeV}$ ions are finding recently wider use in radiation therapy for, e.g., treatment of different forms of cancer. In spite of this, the understanding of the evolution of energy deposited around the path of an ion has not progressed markedly since early works in the sixties and seventies [3-5].

Ions with velocities higher than the Bohr velocity $\left(v_{B}=0.22 \mathrm{~cm} / \mathrm{ns}\right)$ deposit their energy in electronic excitation and ionizations in a cylindrically symmetric region around the path of the impacting ion, forming the track core. The incident ions cause ejection of energetic secondary electrons that transport part of this energy out of the core $[3,4]$. The mean deposited energy density, $e(r)$, after the ion passage is approximated by a $e(r) \propto r^{-2}$ dependence, where $r$ is the radial distance from the ion path. Such a dependence is obtained from theory employing the continuous slowing down approximation $[4,6]$ for the secondary electrons, Monte Carlo simulations [5-7], as well as experiments performed in gases [8,9]. Several energy-transport mechanisms [10,11] have been advanced to describe the subsequent energy propagation and dissipation. However, no direct experimental determination of the initial or evolved energy distribution exists for targets in the condensed phase. In this work a first estimate of $e(r)$, the radial profile of the mean deposited energy density, is made for a solid material.

When fast ions interact with dielectric materials, a trail of radiation damage in the bulk (damage tracks) [3] as well as material erosion of the surface (electronic sputtering) are observed $[12,13]$. Whereas the characteristics of latent damage tracks have been determined by various physical methods ex situ, long after the impacting event [14], the study of the sputtered material can in principle provide "real time," in situ information on the complex physical and chemical processes occurring in ion tracks
[15-17]. Here we demonstrate the use of high resolution time-of-flight mass spectrometry of the ionic ejecta $[13,17]$ to reconstruct the track of an $\mathrm{MeV}$ ion in an organic polymer on the picosecond time scale.

Radial velocity distributions of hydrocarbon secondary ions, ejected from poly(vinylidene fluoride), PVDF $\left(\left[-\mathrm{CH}_{2} \mathrm{CF}_{2}-\right]_{n}\right)$, and other polymer targets have been correlated to their chemical compositions [18]. This is exemplified in Fig. 1, where the mean radial velocities, $\left\langle v_{x}\right\rangle$, and the FWHM $\left(\propto\left\langle v_{x}^{2}\right\rangle\right)$ of the velocity distributions of hydrocarbon ions ejected from a pristine PVDF film are plotted as a function of the ion mass. These moments of the radial velocity distributions are seen to vary systematically with the degree of hydrogenation of the secondary ions. Such an effect demonstrates that there is a correlation between the secondary ion chemical composition and the ion formation and ejection processes. The composition of the ejected positive ions is directly related to their chemical and thermal history in the track. For example, ionic species, e.g., $\mathrm{C}_{n}{ }^{+}$, formed by a strong depletion of $\mathrm{H}$ atoms from the macromolecular chains have the highest $\left\langle v_{x}^{2}\right\rangle$ values (Fig. 1), suggesting these ions originate from the "hotter" (and inner) regions of the track. In contrast, saturated (e.g., $\mathrm{C}_{n} \mathrm{H}_{2 n}{ }^{+}$) species show the lowest $\left\langle v_{x}^{2}\right\rangle$ values. Thus the dependence of $\left\langle v_{x}^{2}\right\rangle$ on ion mass in Fig. 1 actually reflects the radial profile of the deposited energy. These data can, in principle, be used to determine the radial profile of the energy density, as recently argued [18], but determining this would require a spatial model for the ejection process.

Alternatively, having an independent determination of the location of ejection of the ions, the results from Fig. 1 can be converted into radial profiles of the energy density in the track of a single ion. A direct measurement on the nanometer scale of the region in the track from which a particular ion originates is, of course, difficult to realize experimentally. Here we show that an experimental estimate of the mean ejection radius for the secondary ions can be inferred from the "disappearance cross section" $[14,19,20]$. In the context of sputtering, the disappearance cross section is derived from the decrease in the yield of 


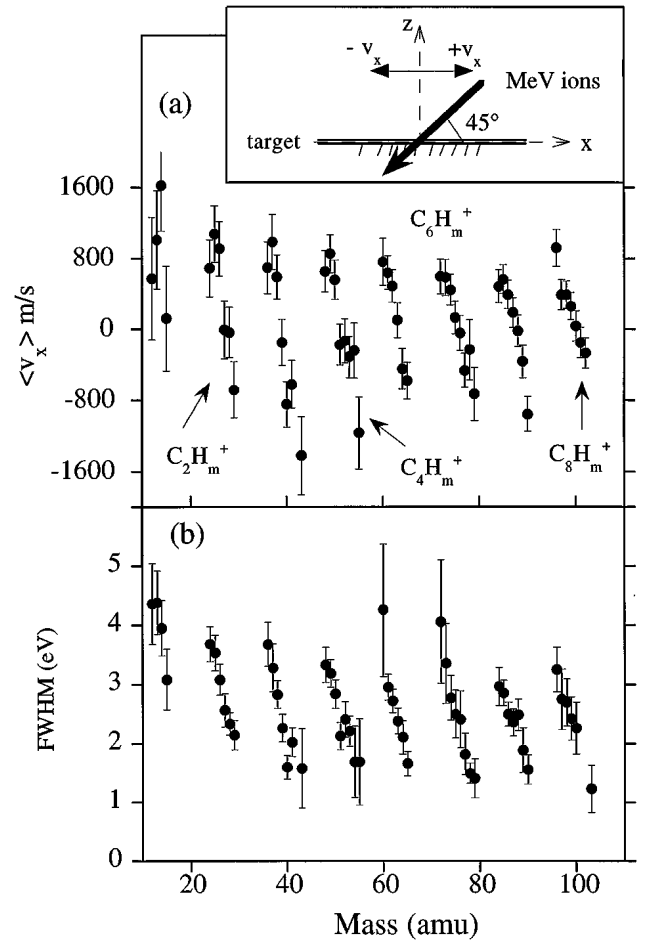

FIG. 1. (a) Mean radial velocities $\left\langle v_{x}\right\rangle$ for the $\mathrm{C}_{n} \mathrm{H}_{m}^{+}$ion series ejected from PVDF by charge-equilibrated $72.3 \mathrm{MeV}$ ${ }^{127}$ I ions. (b) FWHM $\left(=0.5 M\left[\left\langle v_{x}^{2}\right\rangle-\left\langle v_{x}\right\rangle^{2}\right]\right)$ of the $v_{x}$ distributions for the same ions (adapted from Ref. [18]). In these experiments, a high resolution reflectron time-offlight mass spectrometer was used. It provided unequivocal assignment of the secondary ion chemical composition [in the range $(1-100) M / Z]$, based on accurate mass determination. The $x$ and $y$ components of the radial velocity distribution were determined by monitoring the secondary ion yield as a function of the voltage applied to deflection plates parallel to the target normal. For a detailed description of the experimental procedure see Ref. [14]. The instrumental broadening accounts for approximately $40 \%$ of the measured FWHM (Ref. [24]). The signs of the velocities are defined by the coordinate system in the inset (an ion with positive $x$ and $z$ velocities is ejected towards the line of incidence of the $\mathrm{MeV}$ primary ions). We use here the term "radial velocity" for brevity, although only the component of the radial (tangential) velocity in the $x$ direction is reported.

a particular species $Y_{i}$, with irradiation time given as a fluence [20]. If the evolution of $Y_{i}$ with fluence $\phi$ has a simple exponential behavior,

$$
Y_{i}(\phi)=Y_{i 0} \exp \left(-\sigma_{i} \phi\right)
$$

then $\sigma_{i}$ is defined as the disappearance cross section for a polyatomic species $i$.

The quantity $\sigma_{i}$ is an effective area associated with an individual ion impact and a particular ejected ion. Since the ejection of a fragment ion requires the production of damage locally, there is overlap between the region in which an appropriate level of damage is produced and the region from which a particular ion is ejected. For randomly impacting ions, in addition to causing ejection of a fragment, this leads to a decrease in the yield.
This decrease typically follows a Poisson distribution, leading to Eq. (1). Because of the relationship between the production of a fragment and damage, the quantity $\sigma_{i}$ can be associated with an effective ejection area. That is, within the area $\sigma_{i}$ the energy density is too high for an ion of a particular size to be formed and ejected intact. At some larger distance, the energy density will be too low for such ions to be formed and ejected. Thus ejection can be considered to occur from a ring with inner radius, roughly $\left(\sigma_{i} / \pi\right)^{1 / 2}$, and some outer radius $\left(\sigma_{i} / \pi\right)^{1 / 2}+\Delta r$. Assuming that $\Delta r \ll\left(\sigma_{i} / \pi\right)^{1 / 2}$, the effective ejection radius $\bar{r}$ is approximately $\left(\sigma_{i} / \pi\right)^{1 / 2}$. This interpretation of the measured damage cross section is seen below to be consistent with the data on $\left\langle v_{x}^{2}\right\rangle$ and $\left\langle v_{x}\right\rangle$.

The disappearance cross sections for positive secondary ions ejected from PVDF targets, using charge-equilibrated $55 \mathrm{MeV}{ }^{127} \mathrm{I}$ as primary ions, were obtained in the following experiment [21]. A $1 \times 1 \mathrm{~mm}^{2}$ sample area was bombarded at $45^{\circ}$ angle of incidence with a beam current less than $5 \mathrm{nA} / \mathrm{cm}^{2}$ to the desired fluence. Secondary ion mass analysis of the irradiated target was done in situ after each irradiation by reducing the beam intensity to $\approx$ 2000 ions/s. The ejected secondary ions were analyzed in a reflectron time-of-flight mass spectrometer [22]. The ion flight time registration was performed in an event-by-event mode by employing a time-to-digital converter. Typically $4 \times 10^{6}$ primary ion impacts were accumulated for each mass spectrum. The process was repeated by increasing the fluence (from $10^{9}$ to $1.3 \times 10^{13}$ ions $/ \mathrm{cm}^{2}$ ), bombarding one and the same target spot. The vacuum in the experimental chamber was always better than $5 \times 10^{-9}$ torr.

The value of $\sigma_{i}$ for a specific ion is obtained by fitting Eq. (1) to the data points for ion intensity, $Y_{i}(\phi)$, as a function of fluence $\phi$. The $\sigma_{i}$ values obtained for different hydrocarbon ions, $\mathrm{C}_{n} \mathrm{H}_{m}{ }^{+}$, in the range up to $M / Z=100$, where $M$ and $Z$ are, respectively, the ion mass and charge, are shown in Fig. 2. The $\sigma_{i}$ values are seen to exhibit a regular behavior with the ion mass, meaning that $\sigma_{i}$ is correlated with the chemical composition of the ions, as were $\left\langle v_{x}\right\rangle$ and FWHM. The

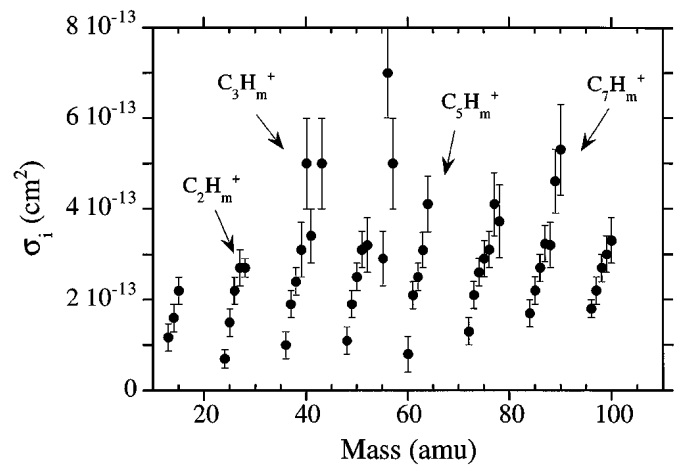

FIG. 2. Disappearance cross sections $\sigma_{i}$ for hydrocarbon secondary ions ejected from PVDF targets by $55 \mathrm{MeV}{ }^{127} \mathrm{I}$, as a function of mass $M$ (see text for further details). 
cross sections, or equivalently the effective ejection radii, increase with increasing number of hydrogen atoms in the ion species for the same $\mathrm{C}$-atom series. This is sensible since the heat of formation of these ions depends on their chemical composition and the degree of fragmentation within the track region depends on the transient local energy density in the track. Therefore, the observed dependence of $\sigma_{i}$ on ion mass indicates the origin of ion ejection. Ions with a lower number of hydrogen atoms originate from the hotter areas of the ion track, thus having both smaller effective ejection radii and higher $\left\langle v_{x}^{2}\right\rangle$. In comparison, ions with higher hydrogen content are formed further away from the track center. Therefore, the three sets of data (the $\left\langle v_{x}\right\rangle,\left\langle v_{x}^{2}\right\rangle$ and $\sigma$ ) are selfconsistent if we use the interpretation above for the damage cross section as an effective ejection radius. A slight increase in $\sigma$ with increasing mass (i.e., size) of the species is also discerned for the pure carbon cluster ions, but the hydrogen content is more sensitive to the local energy density.

The cross sections derived from secondary ion yields also determine the eventual macroscopic transformations of the properties of the targets, such as mechanical and electrical properties, coupled to the carbonization of the polymer under long-term irradiation [21]. Thus the cross sections were estimated excluding the high fluence points. Not surprisingly, the cross section values of $\mathrm{C}^{+}$are close in size to the carbonization cross section $\sigma_{c}$ for the material: i.e., $\sigma_{c} \leq \sigma_{i}$ for these ions, which will give a carbonization radius less than about $8 \AA$ for a single ion. For comparison, we note that the so-called track core radius (the Bohr adiabatic radius) is $\approx 4 \AA$ for $55 \mathrm{MeV}{ }^{127} \mathrm{I}$ ions.

The quantities $\left\langle\boldsymbol{v}_{x}\right\rangle$ and $\left\langle\boldsymbol{v}_{x}^{2}\right\rangle$ are indicative of the momentum transfer to the lattice and the local deposited energy deposited energy density [23] $e(r)$ averaged over the time of ejection. Using the effective radii $\bar{r}$, determined from the disappearance cross section $\sigma_{i}$, these quantities can be coupled to an effective radial distance about the track center for each ejected ionic species. The resulting radial profiles of $\left\langle v_{x}^{2}\right\rangle$ and $\left\langle v_{x}\right\rangle$ are given in Fig. 3. Data for $\mathrm{C}_{n} \mathrm{H}_{m} \mathrm{~F}$ secondary ions, also included in the plots, follow the same trend of the pure hydrocarbon ion series. We note that the velocity distributions were obtained using $72.3 \mathrm{MeV}{ }^{127} \mathrm{I}$ ions, with a slightly higher stopping power $(\approx 10 \%)$, and velocity $(\approx 15 \%)$, compared to $55 \mathrm{MeV}{ }^{127} \mathrm{I}$ ions. This results in a higher mean deposited energy density in the track for $55 \mathrm{MeV}{ }^{127} \mathrm{I}$ ions. The effect on the velocity distributions should be small, as $\left\langle\boldsymbol{v}_{x}^{2}\right\rangle$ and $\left\langle\boldsymbol{v}_{x}\right\rangle$ increase slowly with $d E / d x$, the stopping power [24]. However, previous data on the damage cross section obtained for polymer films indicate an increase in the cross sections of up to a factor of 2 for $55 \mathrm{MeV}$ I bombardments compared to $78 \mathrm{MeV}$ I ions [25]. To account for these differences the radii in Fig. 3 were divided by a factor of $\sqrt{2}$.

The experimental points in Fig. 3(a) are well described by a power law decrease. The best fit to the data is $\left\langle v_{x}^{2}\right\rangle \propto \bar{r}^{-1.2}$, but powers between $r^{-1}$ and $r^{-2}$ also fit

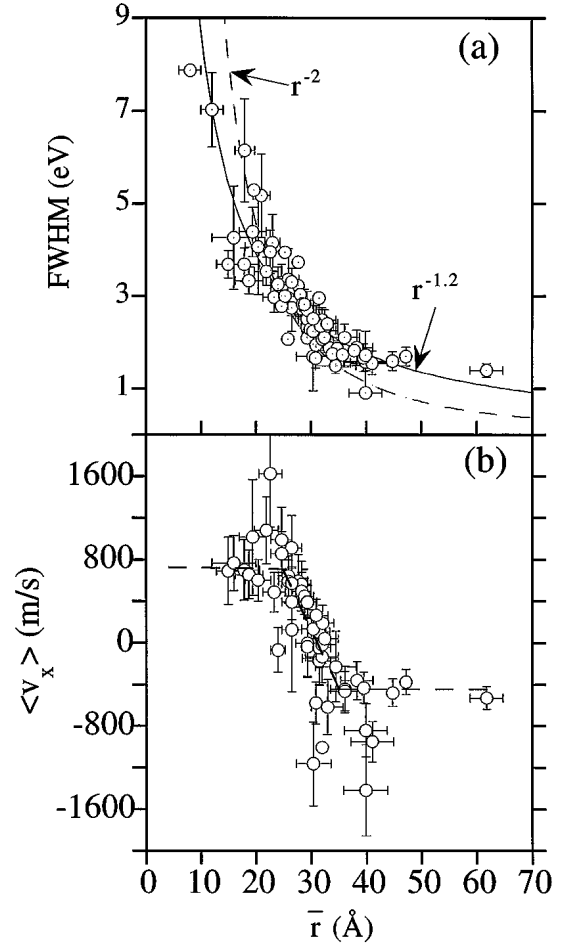

FIG. 3. Profiles for (a) the FWHM $\left(=0.5 M\left[\left\langle v_{x}^{2}\right\rangle-\left\langle v_{x}\right\rangle^{2}\right]\right)$ and (b) the mean velocities $\left\langle v_{x}\right\rangle$ as a function of the effective ejection radius $\bar{r}$. The FWHM, $\left\langle v_{x}\right\rangle$, and $\bar{r}$ values shown were obtained for $\mathrm{C}_{n} \mathrm{H}_{m}{ }^{+}$and $\mathrm{C}_{n} \mathrm{H}_{m} \mathrm{~F}^{+}$ions ejected from PVDF films. For details, see text.

considering the scattering of the data and the uncertainties introduced by the corrections to $\bar{r}$.

In Fig. 3(b), the mean velocity (related to the impulse transferred to the ions) is displayed as a function of the effective radius. The following trends can be distinguished. At small $\bar{r}$ (up to $\approx 25 \AA$ ), ions have a positive $\left\langle v_{x}\right\rangle$ (corresponding to ejection backwards along the track; see inset of Fig. 1), as well as higher $\left\langle v_{x}^{2}\right\rangle$. At large $\bar{r}(>35 \AA)$, ions tend to have similar mean velocities, but away from the line of incidence of the $\mathrm{MeV}$ ion. A relatively steep transition region between 25 and $35 \AA$ is also seen which is roughly consistent with the simple pressure pulse model for electronic sputtering [26]. In Fig. 3(b) the dashed lines mark approximately these three regions. It is seen from Figs. 3(a) and 3(b) that the near-surface energy density in the track produces fragmentation and ejection processes that are closely intertwined. The local energy density triggers the fast "hot" chemistry responsible for bond rearrangement in the track [25]. However, the dependence of the mean velocity of the ions with the place of emission indicates ejection occurs in response to the volume force arising from the energy density gradient in the track [26]. It provides the momentum transfer critical for removing newly formed species from the surface sufficiently rapid for survival. We note that the direction of the momentum transferred to the target species, and thus the variation of $\left\langle v_{x}\right\rangle$ with $\bar{r}$, is coupled to the geometry of the MeV ion impact. For example, in an impact at normal incidence it 
is expected that $\left\langle v_{x}\right\rangle \approx 0$ for the same ions [27], distinct from the dependence found here for $45^{\circ}$ impacts.

In conclusion, we have derived directly from experiments the radial variation of quantities related to the mean energy density $\left(\propto\left\langle v_{x}^{2}\right\rangle\right)$ and the surface momentum transfer $\left(\propto\left\langle v_{x}\right\rangle\right)$ for the track of a polymer film using secondary ion ejection. This was done in a self-consistent way using the damage cross section for the radial scale. This quantitative result can be used as a test for modeling track formation. The following qualitative picture of the track structure is obtained: Species emerging closer to the core (small $\bar{r}$ ) tend to have the largest $\left\langle\boldsymbol{v}_{x}^{2}\right\rangle$ and positive $\left\langle\boldsymbol{v}_{x}\right\rangle$ (towards the line of incidence of the $\mathrm{MeV}$ ions); species characterized with a large $\bar{r}$ have smaller $\left\langle v_{x}^{2}\right\rangle$ and negative $\left\langle v_{x}\right\rangle$ (away from the incoming $\mathrm{MeV}$ ion direction). The radial variation of the mean deposited energy density at the time of ejection obtained here appears to be less steep than the theoretical $r^{-2}$ dependence obtained for the initial energy density in the track of fast ions in a gas. This dependence is in rough agreement with recent modeling of ion tracks in polymers [25].

This work was supported by the Swedish Natural Sciences Research Council (NFR), the Wallenberg Foundation, and the Ångström and Cluster Consortia. M. Inokuti and C.T. Reimann are acknowledged for helpful comments.

*Permanent address: Institute of Physics, Federal University of Rio Grande do Sul, C.P. 15051, 91501-970 Porto Alegre, Brazil.

[1] R. Spohr, Ion Tracks and Microtechnology (Vieweg, Braunshweig, 1990).

[2] Proceedings of the First Conference of Ionizing Radiation and Polymers, Guadeloupe, France, 1994 [Nucl. Instrum. Methods Phys. Res., Sect. B 105 (1995)].

[3] R.L. Fleischer, P. Price, and R.M. Walker, Nuclear Tracks in Solids (Univ. of California Press, Berkeley, 1975).

[4] R. Katz, S. C. Sharma, and M. Homayoonfar, in Topics in Radiation Dosimetry, Supplement 1, edited by F. H. Attix (Academic Press, New York, 1972), p. 317.

[5] G. Kraft, M. Krämer, and M. Scholz, Radiat. Environ. Biophys. 31, 161 (1992).

[6] M.P. Waligórski, R. N. Hamn, and R. Katz, Nucl. Tracks Radiat. Meas. 11, 309 (1986).

[7] H. G. Paretzke, in Kinetics of Nonhomogeneous Processes, edited by G. Freeman (John Wiley \& Sons, New York, 1987), p. 89.

[8] M. N. Varma, J. W. Baum, and A. V. Kuehner, Phys. Med. Biol. 24, 651 (1980).
[9] N. F. Metting, H. H. Rossi, L. A. Braby, P. J. Kliauga, J. Howard, M. Zaider, W. Schimmerling, M. Wong, and M. Rapkin, Radiat. Res. 116, 183 (1988).

[10] R. E. Johnson and W. L. Brown, Nucl. Instrum. Methods Phys. Res. 198, 103 (1982).

[11] C. C. Watson and T. A. Tombrello, Radiat. Eff. 89, 263 (1985).

[12] R.E. Johnson and B. U. R. Sundqvist, Phys. Today 45, No. 3, 28 (1992).

[13] K. Wien, Rad. Eff. Defects Solids 109, 137 (1989); Nucl. Instrum. Methods Phys. Res., Sect. B 65, 149 (1992).

[14] M. Toulemonde, S. Bouffard, and F. Studer, Nucl. Instrum. Methods Phys. Res., Sect. B 91, 108 (1994).

[15] R. D. Macfarlane and D. F. Torgerson, Phys. Rev. Lett. 36, 486 (1976).

[16] W. Ens, B. U. R. Sundqvist, P. Håkansson, A. Hedin, and G. Jonsson, Phys. Rev. B 39, 763 (1989).

[17] G. Brinkmalm, P. Demirev, D. Fenyö, P. Håkansson, J. Kopniczky, and B. U.R. Sundqvist, Phys. Rev. B 47, 7560 (1993).

[18] R. M. Papaléo, G. Brinkmalm, D. Fenyö, J. Eriksson, H.-F. Kammer, P. Demirev, P. Håkansson, and B. U. R. Sundqvist, Nucl. Instrum. Methods Phys. Res., Sect. B 91, 667 (1994).

[19] H. Dertinger and H. Jung, Molecular Radiation Biology (Springer-Verlag, Heidelberg, 1969).

[20] M. Salehpour, P. Håkansson, and B. U. R. Sundqvist, Nucl. Instrum. Methods Phys. Res., Sect. B 2, 752 (1984).

[21] R. M. Papaléo, A. Hallén, P. Demirev, G. Brinkmalm, J. Eriksson, P. Håkansson, and B. U.R. Sundqvist, Nucl. Instrum. Methods Phys. Res., Sect. B 91, 677 (1994).

[22] G. Brinkmalm, P. Håkansson, J. Kjellberg, P. Demirev, B. U.R. Sundqvist, and W. Ens, Int. J. Mass Spectrom. Ion Process. 114, 183 (1992).

[23] The total energy is related to the kinetic energy $\left(\left\langle v_{x}^{2}\right\rangle\right)$ plus the chemical energy of rearrangement and fragmentation reactions. The Coulomb potential energy "stored" in the electric field of the localized ionic species in the track core may also contribute to the $\left\langle v_{x}^{2}\right\rangle$ value by, e.g., accelerating the positive ions during ejection.

[24] R. M. Papaléo, P. Demirev, J. Eriksson, P. Håkansson, and B. U. R. Sundqvist, Phys. Rev. B (to be published).

[25] R. M. Papaléo, L. Farenzena, A. Hallén, M. A. D. Araújo, R. P. Livi, B. U.R. Sundqvist, and R.E. Johnson, Phys. Rev. B 53, 2303 (1996).

[26] R.E. Johnson, B. U.R. Sundqvist, A. Hedin, and D. Fenyö, Phys. Rev. B 40, 49 (1989); D. Fenyö, ibid. 47, 8263 (1993).

[27] D. Fenyö, A. Hedin, P. Håkansson, and B. U. R. Sundqvist, Int. J. Mass Spectrom. Ion Process. 100, 63 (1990). 